\title{
Rare Recurrence of Hepatocellular Carcinoma Greater than 6 Years after Living Donor Liver Transplant
}

\author{
Nha T Duong ${ }^{1}$, Arish Noor ${ }^{1}$ and Martin Hoffman² \\ ${ }^{1}$ Department of Internal Medicine, University of Connecticut School of Medicine, USA \\ ${ }^{2}$ Department of Gastroenterology and Hepatology, Yale University School of Medicine, USA
}

Submission: October 01, 2018; Published: October 10, 2018

${ }^{*}$ Corresponding author: Nha T Duong, Department of Internal Medicine, University of Connecticut, School of Medicine, USA, Email: nhduong@uchc.edu

Keywords: Liver transplant; Gross vascular; Liver Diseases; Pneumonia; Orthotopic liver; Abdominal; Chest

Abbreviations: LDLT: Living Donor Liver Transplant; HCC: Hepatocellular Carcinoma; OLT: Orthotopic Liver Transplant; LT: Liver Transplant; DDLT: Donor Liver Transplant; AASLD: American Association for the Study of Liver Diseases; AFP: Alpha Fetoprotein Level; RFA: Radiofrequency Ablation; TACE: Transarterial Chemoembolization

\section{Introduction}

Living donor liver transplant (LDLT) for patients within Milan criteria (one lesion smaller than $5 \mathrm{~cm}$; up to 3 lesions smaller than $3 \mathrm{~cm}$, no extrahepatic manifestations, no evidence of gross vascular invasion) has been an evolving modality for the treatment of hepatocellular carcinoma (HCC) in the last decade $[1,2,3]$. Orthotopic liver transplant (OLT) was once considered an experimental approach in the pediatric population has now gained its popularity in the management of liver cancer in adults and has led to shortening of time on the liver transplant (LT) waitlist. Observational studies have shown comparable outcomes between LDLT and deceased donor liver transplant (DDLT) with regards to overall survival and disease-free survival rates. Unfortunately, recurrence post LDLT as with DDLT remains an ongoing issue and rates up to $10 \%$ have been reported at 4 years post-transplant [4]

There are no specific guidelines for surveillance after liver transplant (LT) including the choice of surveillance method, or interval and duration of surveillance. A reasonable proposal by the American Association for the Study of Liver Diseases (AASLD) in the 2012 practice guideline of long-term management of successful adult liver transplant is to continue surveillance with abdominal and chest computed tomography (CT) every 6 months for 3 years after LT, with closer attention dedicated to those with elevated alpha fetoprotein level (AFP) [5,6]. Current guidelines define that recurrence-free survival after 5 years is considered curative treatment. We herein describe a rare, and unfortunate, case of HCC recurrence greater than 6 years after LDLT found incidentally on a chest CT for pneumonia follow-up.

\section{Case Report}

The patient is a 69-year-old male with an extensive past medical history of chronic hepatitis $\mathrm{B}$ and hepatocellular carcinoma (HCC) that presented to our facility as a follow up for abnormal imaging findings on recent CT scan. His medical history dated back to 2007 when he was diagnosed with an HCC in the right hepatic lobe. The mass was resected shortly after diagnosis, but unfortunately the patient had recurrence of HCC in 2011 associated with his chronic hepatitis B infection and was evaluated for transplant. He underwent successful orthotopic liver transplant with his daughter as a living donor to the right hepatic lobe. The patient was placed on immunosuppressant therapy with mycophenolate and cyclosporine.

He has been on surveillance for hepatocellular carcinoma with annual abdominal imaging and alpha-fetoprotein (AFP) levels. The patient had an MRI in 2016, 5-years post-transplant, which showed no liver masses and patent graft vessels. With regards to his hepatitis $B$, the patient had been on treatment with tenofovir. His labs in 2016 showed an undetectable quantitative hepatitis B polymerase chain reaction, negative hepatitis B surface antigen, and AFP level of 2.6 (units). Towards the end of 2017 the patient was admitted to the hospital for respiratory distress and diagnosed with pneumonia on chest CT. Repeat imaging for follow up of his pneumonia in early 2018 showed an incidental finding of multiple hyperdense and hypodense lesions throughout the liver parenchyma concerning for hemorrhagic metastatic disease. 
A follow-up MRI was done ( $>6$ years post-transplant) to characterize the lesions and showed multiple hemorrhagic masses within the transplanted liver concerning for recurrent HCC. The largest of these masses measured up to $6.8 \mathrm{~cm}$. He underwent CT guided biopsy of the right hepatic lobe, which showed benign liver parenchyma with focal necrosis and organizing clot but no evidence of malignancy. Due to a concerning clinical picture for recurrent disease, a recommendation was made for repeat biopsy of segment 2 and 3, which unfortunately resulted as moderately differentiated hepatocellular carcinoma. The patient was diagnosed with multifocal recurrent HCC. Hepatitis labs revealed positive hepatitis $B$ surface antigen, negative hepatitis $B$ surface antibody, and detectable viral load for the first time since transplant indicating active infection, likely recurrence. His AFP level remained normal at 4.1. Due to the multitude of his disease the patient was not a candidate for surgery, radiofrequency ablation (RFA), or transarterial chemoembolization (TACE). He is currently being evaluated for local regional therapy with lobar yttrium-90 (Y-90) radio embolization by intervention radiology.

\section{Discussion}

With recent advancements, liver transplantation has evolved as potential therapy with intent to cure hepatocellular carcinoma. In a landmark study by Mazzaferro et al. [1] in 1996, it was shown that specific criteria for selecting cases of cirrhosis with HCC for LT led to improved overall and disease-free survival at the 4-year time frame [1]. Results of this study later served as the basis for the Milan criteria. Similarly, Yao et al. from the University of California San Francisco (UCSF) later reported survival in $75 \%$ of patients at the 5-year post-transplant time frame [2]. In the UCSF study, selection criteria for transplantation included: tumors up to $6.5 \mathrm{~cm}$, or up to 3 lesions each being less than $4.5 \mathrm{~cm}$ with total tumor burden less than $9 \mathrm{~cm}$. The current Organ Procurement and Transplantation Network (OPTN)/ONUS guidelines approve consideration for liver transplant in patients with cirrhosis and HCC who meets the aforementioned Milan criteria [3].

LDLT has made transplantation more readily available and decreased the time on the transplant waitlist for many patients [4]. Data regarding its survival and recurrence has been limited as the procedure only gained popularity within the past decade. A 2012 study by Sandhu et al. looked at the overall survival (OS) rates and rates of HCC recurrence post-transplant between a total of 345 patients who received LDLT and DDLT. There were no differences between the rate of recurrence and OS between the two groups at 1,3 , ad 5 years post-transplant. Their overall survival was reported as $75.2 \%$ in the LDLT group and $74.6 \%$ in the DDLT group with p-value showing no statistical significance. 3-year post transplant HCC recurrence was determined to be $10.7 \%$ in the LDLT group and $14.8 \%$ in the DDLT group, also with no statistical significance [7]. Rates of OS and recurrence-free survival at 5-years post-transplant have been reported as high as $89.9 \%$ and $97.0 \%$, respectively, in a similar study [8]. These findings show promising data for LDLT as a modality for treatment of HCC with intent to improve mortality.
Recurrence of HCC after transplant poses a difficult clinical situation that is rarely curable and is associated with high mortality. Multiple studies have drawn similar conclusions to the risk factors for recurrence of HCC post-transplant. In observational studies, it was noted that elevated neutrophil to lymphocyte ratio (NLR) and AFP levels (>200 ng/mL) were found in patients who demonstrated recurrence of disease post-transplant [8].

A 2011 study by Nissen et al. noted recurrence in 13 of 122 patients with a median time frame of 14 months post-transplant. Univariate analysis associated bilobar tumors, vascular invasion, elevated levels of PIVKA-II, and stage exceeding Milan and UCSF criteria as predictors for recurrence [9-11]. Interesting enough, our patient had consistently low levels of AFP as well as neutrophil to lymphocyte ratio during his surveillance period. Also, at the time of initial diagnosis, he did not have bilobar invasion or staging that exceeded Milan criteria. The lack of risk factors may have explained the delayed recurrence in our patient at $>6$ years post-transplant yet, the dire question of how remains.

LDLT and HCC recurrence has been correlated with several significantly elevated serum biomarkers. Chiu et al. demonstrated that levels of fibroblast growth factor (FGF-2), survivin, Ki67, endostatin, and vascular endothelial growth factor (VEGF) were elevated in patients with recurrent HCC after LDLT who also demonstrated microvascular invasion [12]. These findings may be implicated in the pathogenesis of recurrence but larger studies with longer follow up would be required for confirmation. A new theory regarding the existence of cancer stem cells in HCC has been proposed but its understanding is limited [13]. Since our patient received his donor liver from his daughter, it would be interesting to find out the origin of the recurrent tumor by identifying the presence of sex chromosomes in the tumor cells.

Recurrence of HCC after LDLT poses a high mortality risk and options for management remains limited. If the tumor size is amendable and there is no vascular invasion, surgical resection would be the ideal treatment of choice. Systemic treatment with an mTOR inhibitor and sorafenib has been proposed, but data regarding its efficacy is limited [14]. Nonetheless, it may be beneficial to aim studies at determining the pathogenesis of recurrence to provide better insight into the disease itself. Currently, not enough evidence is available to propose high quality recommendations into surveillance post-transplant. By identifying rare cases of recurrence, such as our patient with limited risk factors, we question the need for prolong posttransplant surveillance, possibly beyond 5-years.

\section{References}

1. Mazzaferro V, Regalia E, Doci R, Andreola S, Pulvirenti A, et al. (1996) Liver Transplantation for the Treatment of Small Hepatocellular Carcinomas in Patients with Cirrhosis. N Engl J Med 334(11): 693-700.

2. Yao FY, Ferrell L, Bass NM, Watson JJ, Bacchetti P, et al. (2001) Liver transplantation for hepatocellular carcinoma: Expansion of the tumor size limits does not adversely impact survival. Hepatology 33(6): 13941403. 
3. Martin P, DiMartini A, Feng S, Brown R Jr, Fallon M (2014) Evaluation for Liver Transplantation in Adults: 2013 Practice Guideline by the American Association for the Study of Liver Diseases and the American Society of Transplantation. Hepatology 59(3): 1144-1165.

4. Rahimi RS, Trotter JF (2015) Liver transplantation for hepatocellular carcinoma: outcomes and treatment options for recurrence. Ann Gastroenterol 28(3): 323-330.

5. Lucey MR, Terrault N, Ojo L, Hay JE, Neuberger J, et al. (2013) Long-Term Management of the Successful Adult Liver Transplant: 2012 Practice Guideline by AASLD and the American Society of Transplantation. Liver Transpl 19(1): 3-26.

6. Heimbach JK, Kulik LM, Finn RS, Sirlin CB, Abecassis MM, et al. (2018) AASLD Guidelines for the Treatment of Hepatocellular Carcinoma. Hepatology 67(1): 358-380.

7. Sandhu L, Sandroussi C, Guba M, Selzner M, Ghanekar A, et al. (2012) Living donor liver transplantation versus deceased donor liver transplantation for hepatocellular carcinoma: comparable survival and recurrence. Liver Transpl 18(3): 315-322.

8. Yoshizumi T, Harimoto N, Itoh S, Okabe H, Kimura K, et al. (2016) Living Donor Liver Transplantation for Hepatocellular Carcinoma within Milan Criteria in the Present Era. Anticancer Res 36(1): 439-445.
9. Nissen NN, Menon V, Bresee C, Tran TT, Annamalai A, et al. (2011) Recurrent hepatocellular carcinoma after liver transplant: identifying the high-risk patient. HPB (Oxford) 13(9): 626-632.

10. Abdel-Wahab M, Sultan AM, Fathy OM, Salah T, Elshobary MM, et al. (2013) Factors affecting recurrence and survival after living donor liver transplantation for hepatocellular carcinoma. Hepatogastroenterology 60(128): 1847-1853.

11. Choi HJ, Kim DG, Na GH, Han JH, Hong TH, et al. (2013) Clinical outcome in patients with hepatocellular carcinoma after living-donor liver transplantation. World J Gastroenterol 19(29): 4737-4744.

12. Chiu KW, Nakano T, Chen KD, Hsu LW, Lai CY, et al. (2015) Repeatedmeasures implication of hepatocellular carcinoma biomarkers in living donor liver transplantation. PLoS One 10(5): e0124943.

13. Kanno Y, Takahashi A, Abe K, Takagi T, Okai K, et al. (2012) Hepatocellular Carcinoma that Relapsed 54 Months after Living Donor Liver Transplantation. Intern med 51(17): 2325-2328.

14. Mazzola A, Constantino A, Petta S, Bartolotta TV, Raineri M, et al. (2015) Recurrence of hepatocellular carcinoma after liver transplantation: an update. Future Oncol 11(21): 2923-2936.

\section{Your next submission with JuniperPublishers} will reach you the below assets

- Quality Editorial service

- Swift Peer Review

- Reprints availability

- E-prints Service

- Manuscript Podcast for convenient understanding

- Global attainment for your research

- Manuscript accessibility in different formats

( Pdf, E-pub, Full Text, audio)

- Unceasing customer service

Track the below URL for one-step submission https://juniperpublishers.com/online-submission.php 